\title{
CONSTRAINTS ON THE ENERGY CONTENT OF THE UNIVERSE FROM A COMBINATION OF GALAXY CLUSTER OBSERVABLES
}

\author{
SANDOR M. MOLNAR ${ }^{1}$, ZOLTÁN HAIMAN ${ }^{2}$, MARK BIRKINSHAW ${ }^{3}$ AND RICHARd F. MUSHOTZKY ${ }^{4}$
}

Draft version July 31,2003

\begin{abstract}
We demonstrate that constraints on cosmological parameters from the distribution of clusters as a function of redshift $(d N / d z)$ are complementary to accurate angular diameter distance $\left(D_{A}\right)$ measurements to clusters, and their combination significantly tightens constraints on the energy density content of the Universe. The number counts can be obtained from X-ray and/or SZ (Sunyaev-Zel'dovich effect) surveys, and the angular diameter distances can be determined from deep observations of the intra-cluster gas using their thermal bremsstrahlung $\mathrm{X}$-ray emission and the $\mathrm{SZ}$ effect. We combine constraints from simulated cluster number counts expected from a $12 \mathrm{deg}^{2} \mathrm{SZ}$ cluster survey and constraints from simulated angular diameter distance measurements based on the X-ray/SZ method assuming a statistical accuracy of $10 \%$ in the angular diameter distance determination of 100 clusters with redshifts less than 1.5 . We find that $\Omega_{m}$ can be determined within about $25 \%, \Omega_{\Lambda}$ within $20 \%$, and $w$ within $16 \%$. We show that combined $d N / d z+D_{A}$ constraints can be used to constrain the different energy densities in the Universe even in the presence of a few percent redshift dependent systematic error in $D_{A}$. We also address the question of how best to select clusters of galaxies for accurate diameter distance determinations. We show that the joint $d N / d z+D_{A}$ constraints on cosmological parameters for a fixed target accuracy in the energy density parameters are optimized by selecting clusters with redshift upper cut-offs in the range $0.5 \lesssim z \lesssim 1$.
\end{abstract}

Subject headings: cosmological parameters - cosmology: theory - galaxies:clusters: general

\section{INTRODUCTION}

A point often raised in the era of "precision cosmology" is that any particular method to determine fundamental cosmological parameters will suffer from degeneracies: only a combination of parameters can be determined accurately (Bridle et al. 2003). This issue has been highlighted recently by a number of papers focusing on the cosmological usefulness of galaxy clusters (Ettori, Tozzi \& Rosati 2003; Vikhlinin et al. 2003; Mei \& Bartlett 2003; Kujat et al. 2002; Newman et al. 2002; RubinoMartin \& Sunyaev 2002; Verde, Haiman \& Spergel 2002; for a recent review based on X-ray observations see Rosati, Borgani \& Norman 2002). Indeed, to date the tightest constraints have been derived using a combination of different methods, as demonstrated recently by the Wilkinson Microwave Anisotropy Probe (WMAP) team (Spergel et al. 2003). The importance of using independent methods to determine cosmological parameters is two-fold: (1) the methods may be combined to break degeneracies and better constrain individual parameters; and (2) consistency tests are possible and systematic errors can be studied.

Although we focus on constraints from the change in the number density of clusters as a function of redshift, $d N / d z$, and from measurements of the angular diameter distance of clusters based on the Sunyaev-Zel'dovich (SZ) effect, $D_{A}$, further cluster observables could be used as additional indicators of cosmological parameters. Levine et al. (2002) showed that using the cluster temperature function to constrain cosmological parameters can be made more efficient by adding the mass-temperature relation normalization from $\mathrm{X}$-ray observations, and discussed

\footnotetext{
${ }^{1}$ Department of Physics and Astronomy, Rutgers University, 136 Frelinghuysen Road, Piscataway, NJ 08854

2 Department of Astronomy, Columbia University, 550 wes! 120th Street, New York, NY 10027

${ }^{3}$ Department of Physics, Bristol University, Tyndall Avenue, Bristol, BS8 1TL, UK

${ }^{4}$ NASA/Goddard Space Flight Center, Laboratory for High Energy Astrophysics, Greenbelt, MD 20771
}

briefly how to optimize cluster surveys based on their method. Majumdar \& Mohr (2003) emphasized that the mass-temperature relation with masses determined in a relatively modest followup program can be used in combination with the $d N / d z$ test to greatly improve systematic limitations that arise from cluster evolution. Several other observables can be useful, such as scaling relations (Bialek et al. 2001; Verde et al. 2002), the shape of the cluster mass function ( $\mathrm{Hu} \mathrm{2003);} \mathrm{and} \mathrm{the} \mathrm{three-dimensional}$ cluster power spectrum (Refregier et al. 2002).

Constraints on $w$ as a function of redshift from planned SZ and supernovae surveys, and from their combination were studied by Weller, Battye \& Kneissl (2002) and Weller \& Albrecht (2002). Note, however, that cosmic variance can also be important in some cluster surveys (Hu \& Kravtsov 2003).

The redshift distribution of the number of clusters, $d N / d z$, is sensitive to both the change of the cosmological volume element and to the growth function of structure formation. Cluster number counts as a function of redshift can be determined from X-ray or SZ surveys (e.g. Carlstrom et al. 2002; Rosati et al. 2002). Haiman, Mohr \& Holder (2001) discussed in detail constraints on dark energy (via the equation of state parameter, $w)$ from the redshift distribution of clusters from future $\mathrm{SZ}$ and $\mathrm{X}$-ray surveys and suggested that combining those constraints with constraints using Type Ia SNe or CMB fluctuations can lift degeneracies in the $\Omega_{m}-w$ plane (see also Wang \& Steinhardt 1998). Holder, Haiman \& Mohr (2001) used the redshift distribution of clusters from future SZ and X-ray surveys to constrain $\Omega_{m}$ and $\Omega_{\Lambda}$ pointing out that, similarly to the parameter space $\left(\Omega_{m}, w\right)$, constraints from cluster redshift distribution are complementary to constraints from Type Ia SNe and CMB fluctuations. The X-ray thermal bremsstrahlung emission and SZ effect (Sunyaev \& Zel'dovich 1980) depend on different combinations of the physicai parameters of the ciuster and cosmology and provide us with a way to determine the angular diameter distance, $D_{A}$, to the cluster (the so-called X-ray/SZ method, for recent, detailed references, see Carlstrom, Holder \& Reese 2002 and Reese et al. 2003). The angular diameter distance 
probes directly the curvature of the Universe.

Molnar, Birkinshaw \& Mushotzky (2002) discussed constraints on models with parameters $\left(\Omega_{m}, w, h\right)$, and $\left(\Omega_{m}, \Omega_{\Lambda}, h\right)$ using simulations of angular diameter distance measurements to clusters of galaxies and showed that the degeneracies in cosmological parameters from this technique are similar to those from $\mathrm{SNe}$ Ia, and therefore they are complementary to constraints from redshift distribution of clusters. This is not surprising, since the luminosity distance, which is utilized in the SN studies, is closely related to the angular diameter distance.

In this paper, we show that it will be possible using clusters of galaxies alone to constrain accurately the matter density parameter, $\Omega_{m}$, and the cosmological constant density parameter, $\Omega_{\Lambda}$, or the equation-of-state parameter, $w=p / \rho$, by combining $d N / d z$ and $D_{A}$ measurements of clusters using the X-ray/SZ method, and estimate the accuracy achievable in these parameters.

Since long observations are needed for high accuracy distance measurements with present day observatories, a relatively small sample of clusters has to be selected. This cluster sample does not have to be assembled from the same survey that is used for the cluster redshift distribution test. Any sufficiently accurate survey would suffice as long as it has the necessary redshift coverage. The question naturally arises: How should we select clusters of galaxies for accurate angular diameter distance determinations? As an example, we discuss how we can optimize the selection of clusters for detailed distance measurements by allowing both the number of clusters in the sample and the upper redshift cut-off of the sample to be free parameters.

The rest of this paper is organized as follows. In $\S 2$, we briefly summarize our methodology. In $\$ 3$, we apply our technique to future cluster samples, and derive constraints on cosmological parameters. In $\S 4$, we discuss the issue of the optimal selection of clusters for angular diameter distance measures. Finally, in $\$ 5$, we offer our conclusions and summarize the implications of this work.

\section{METHODOLOGY}

We have studied combined constraints from the redshift distribution of clusters and angular diameter distance measurements in two different sets of models. Both sets are described by the usual parameters $\left(\Omega_{m}, \Omega_{\Lambda}, w, h, \sigma_{8}, n\right)$, and we choose as our fiducial model $\left(\Omega_{m}=0.3, \Omega_{\Lambda}=0.7, w=-1, h=0.65, \sigma_{8}=\right.$ $0.9, n=1)$. In Model $\mathrm{A}$, we assume a cosmological constant $(w=-1)$, but allow $\left(\Omega_{m}, \Omega_{\Lambda}\right)$ to vary independently. In Model B, we assume the universe is spatially flat $\left(\Omega_{m}+\Omega_{\Lambda}=1\right)$, but allow $w$ to vary. We used constraints from previous simulations based on the redshift distribution of clusters derived from a deep 12 $\operatorname{deg}^{2}$ SZ survey (see Holder et al. 2001, Model A; and Haiman et al. 2001, Model B). We then determined the expected constraints on the same parameters from $D_{A}$ measurements with fixed accuracy, changing the total number of clusters and the upper redshift cutoff of the sample (for a detailed description of the method see Molnar et al. 2002).

Most models of the X-ray emission from clusters of galaxies are based on the assumption of hydrostatic equilibrium. Detailed, spatially resolved X-ray spectroscopy can be used to observe the projected temperature and X-ray surface brightness distribution of the cluster which, combined with the assumption of hydrostatic equilibrium, can be used to determine the physical model (de-projected temperature and gas density distribution, etc.) for the cluster (see for example Evrard et al. 2002). Recent XMM imaging spectra (Pratt \& Arnaud 2003) support previous observations of clusters which show that outside the central regions a large fraction of clusters are roughly isothermal and that the surface density can be described by a $\beta$ model (Jones \& Forman 1984; Mohr, Mathiesen \& Evrard 1999), where the gas density can be written as $\rho(r)=$ $\left(1+\left(r / r_{c}\right)^{2}\right)^{-3 \beta / 2}$. The core radius, $r_{c}$, sets the scale of the cluster atmosphere and $\beta$ specifies the slope of the density profile at large radius (Cavaliere \& Fusco-Femiano 1976). For the sake of concreteness, we here assume an isothermal $\beta$ model for the intra-cluster gas, however this assumption is not essential in the determination of $D_{A}$. The parameters that we need are: (1) $r_{\varepsilon}$ and $\beta$, which define the spatial structure of the intra-cluster gas and can be deduced from X-ray or SZ images; (2) the amplitude of the X-ray brightness and SZ decrement/increment (depending on the observing wavelength); (3) the temperature of the intra-cluster gas and the X-ray absorbing column density; and (4) the redshift of the cluster (derivable from $x$-ray or optical observations).

The most important known redshift independent systematic errors in determining angular diameter distances arise from calibration errors for the SZ and X-ray instruments, and finite cluster sizes from modeling the intra-cluster gas. Calibration errors cause systematic scale errors in the peak intensities of the $\mathrm{SZ}$ effect and X-ray surface brightness. The calibration errors are about $2.5 \%$ for interferometric observations (Carlstrom et al. 2002), and about $10 \%$ errors for XMM-Newton and Chan$d r a$ which induce $5 \%$ and $10 \%$ errors in $D_{A}$ The $\beta$ model may give a divergent cluster mass if not truncated at some finite upper cut off radius. Assuming a $\beta$ model with infinite extent introduces a systematic underestimate of $D_{A}$. Based on numerical simulations, Inagaki et al. (1995) estimated this systematic error to be as large as $10-20 \%$. Systematic error estimates based on observations are much lower (up to 6\%, Holzapfel et al. 1997; Birkinshaw \& Hughes 1994). However, in this paper we focus on the energy density and equation of state parameters which are not sensitive to redshift independent systematic errors since they depend only on the shape of the angular diameter distance-redshift function (for a detailed analysis see Molnar et al. 2002).

Systematic errors depending on redshift do change the shape of the $D_{A}-z$ relation, and therefore could bias the inferred energy density parameters. The most important such candidate is small scale gas clumping that varies with redshift. A general discussion of the effect of clumping on the determination of angular diameter distance can be found in Reese et al. (2003) and Molnar et al. (2002), and references therein. Numerical simulations show that clumping caused by accretion shocks and mergers results in an overestimate in the angular diameter distance to clusters by about $10 \%$ (LCDM, Mathiesen, Evrard \& Mohr 1999). We will ignore this possibility here and simply note that (1) at present, there is no observational evidence for redshift dependent clumping, and (2) it is likely that a redshift depcndent systematic cffect would cause different systematic errors in $d N / d z$ and $D_{A}$ measurements, and thus these kind of errors could be identified based on consistency.

We assume further that the main known random error in determining, $D_{A}$, the orientation bias for non-spherical clusters, has been solved for our sample using galaxy velocity dispersion measurements, or a combination of X-ray, SZ, and weak lensing data with the assumption of hydrostatic equilibrium (Fox \& Pen 2002). While this is an optimistic assumption, we find that 
in a sample of 100 clusters, this effect would inflate our error estimates by only an additional 5\%. We expect about an 5\% error in the cluster parameters from uncertainty in the spatial fitting, and a 5\% error in the X-ray temperature is achievable with XMM-Newton and Chandra (Pratt \& Amaud 2003). For a detailed analysis of the error budget of the X-ray/SZ method, see Molnar et al. (2002). We assume an additional 5\% statisti$\mathrm{cal}$ error from other sources. Finally we assume that the Hubble constant is determined from other measurements.

Fox \& Pen (2002), using numerical simulations, demonstrated that distances to clusters can be determined with a random error of about $5 \%$ combining X-ray, SZ, and lensing measurements and the assumption of hydrostatic equilibrium. Although they ignored errors due to lensing, their result suggest that a $10 \%$ error in the determination of angular diameter distances is realistic in the near future. Therefore we performed simulations using a $10 \%$ error in the $D_{A}$ values. Note, however, that larger errors, as long as they are truly random, can be compensated with using more clusters, for example, assuming a $20 \%$ random error in $D_{A}$, we would need a sample of about 200 clusters, and our final conclusion of this paper would be unaltered. In all of our simulations the redshift distribution was derived by randomly sampling a uniform distribution in redshift space with an upper cut-off at fixed redshift. The number of sampled clusters in the redshift range $0.1-1.5$ changes at most by a factor less than 3 as a function of cosmology, which, in practice, makes it easy to choose a quasi-uniform sample. Note, however, that our conclusions are not sensitive to the exact distribution of the number of clusters in redshift as long as they smoothly cover the targeted redshift range.

Systematic effects in the cluster redshift distribution from the mass-X-ray temperature, the power spectrum normalization, $\sigma_{8}$, and the mass function were studied by Battye \& Weller (2003). They concluded that these systematic effects are important and more studies are necessary to reduce them. Systematic effects in the cluster redshift distribution in SZ surveys from the surface brightness bias and cluster spatial orientation have not been discussed in detail. The detectibility is a function of signal/noise and, for extended sources (like clusters), this is determined by surface brightness and not by flux. X-ray and $S Z$ surveys will not detect very extended clusters which have surface brightness too low to yield the necessary signal/noise. Even if we use a mass-size relation and a constant mass cut off, there is a dispersion around any observed or theoretical masssize relation.

As a result of the orientation bias, prolate clusters will be over-represented since they have larger central SZ and X-ray surface brightnesses than oblate clusters. Faint ellipsoidal clusters aligned with their long axis in the plane of the sky might not be detected, lower mass clusters with their long axis in the LOS will be falsely detected in a given mass bin. However, there are more low mass than high mass clusters, so we underestimate the number of clusters at the given mass threshold. Using a mass cut-off which is higher than the nominal detection-limitimplied mass cut-off would give us a chance to estimate this bias.

We used the likelihood function obtained by Holder et al. (2001) for model parameters $\Omega_{m}$ and $\Omega_{\dot{\Lambda}}$ (our Model A). Holder et al. defined their likelihood function based on the Cash $C$ statistic (Cash 1979), kept $h$ constant, and marginalized over the power spectrum normalization, $\sigma_{8}$. We used the likelihood function of Haiman et al. (2001) for the cluster redshift distribu-

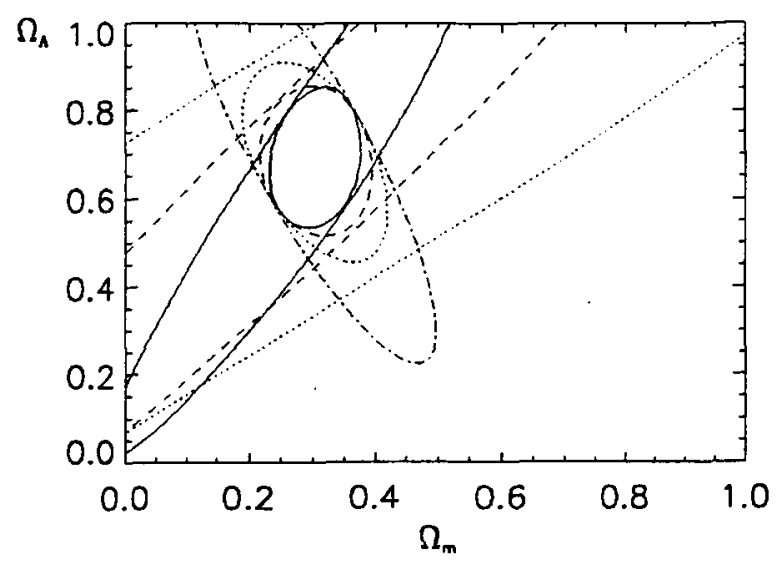

FIG. 1.- Simulated $97 \%$ confidence limits in the $\Omega_{m}-\Omega_{\Lambda}$ plane based on $d N / d z$ and $D_{A}$ measurements. The outer set of solid, dashed, and dotted lines are constraints from the $D_{A}$ values alone for samples of clusters with upper redshift cutoffs of $z=1.5,1.0$, and 0.5 . The dot-dashed line is the constraint from the $d N / d z$ measured in a $12 \mathrm{deg}^{2} \mathrm{SZ}$ survey alone. The corresponding combined confidence intervals are shown as the inner solid, dashed, and dotted curves.

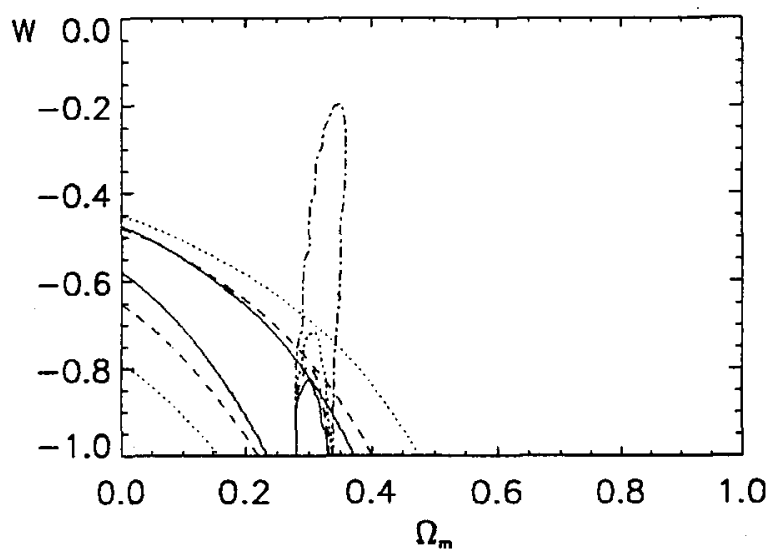

FIG. 2.- Simulated $97 \%$ confidence limits in the $\Omega_{m}-w$ plane based on $d N / d z$ and $D_{A}$ measurements. The line codes are the same as in Figure 1.

tion test in parameter space $\Omega_{m}$ and $w$ (our Model B). Haiman et al. defined their likelihood function as the product of the Poisson probability of detecting a total number of $N$ clusters and a Kolmogorov-Smirnov probability for the unnormalized redshift distribution of these $N$ clusters ${ }^{5}$. Haiman et al. also kept $h$ constant and marginalized over the power spectrum normalization. We kept $h$ constant, assumed Gaussian errors in the diameter distance measurements, and constructed the likelihood function based on the usual $\Delta \chi^{2}$ statistic. The angular diameter distance function does not depend on the power spectrum, so we can combine these two likelibood functions in a straightforward way by multiplying them. In more detailed work on specific observational strategies it would be necessary to multiply the likelihood functions before marginalizing over unconstrained parameters.

\footnotetext{
${ }^{5}$ Note that using the likelihood function based on the Cash statistic would result in somewhat tighter constraints (as demonstrated explicitly by a comparison of these two different statistics in Verde et al. 2001).
} 


\section{COMBINED CONSTRAINTS ON COSMOLOGICAL PARAMETERS}

In our first set of simulations, we assumed 100 clusters with an error of $10 \%$ in the $D_{A}$ determinations with different redshift cut-offs out to $z=1.5$. We show the $97 \%$ confidence limits (CLs) for the combined constraints from the redshift distribution of clusters and angular diameter distance measurements based on simulations in Figures 1 and 2 . In Figure 1 we show constraints on $\Omega_{m}$ and $\Omega_{\Lambda}$ (Model A). This figure shows that, as we select clusters with higher and higher redshift cut-offs, the constraints from $D_{A}$ measurements alone get tighter and the confidence contours rotate counter-clockwise. The constraints get tighter because at higher redshifts, $D_{A}(z)$ is more sensitive to these parameters, and rotate, since these parameters are sensitive to different combinations of the density parameters at different redshifts. As a consequence of this rotation, although at higher upper redshift cul-offs the constraints from $D_{A}$ measurements are tighter, they are farther from orthogonal to constraints from the redshift distribution of clusters. The combined constraints do not improve substantially from upper cut-offs of about $z \gtrsim 1$, although the likelihood function does get somewhat narrower.

We conclude that, contrary to naive expectations, observations of clusters at redshifts exceeding $z \approx 1$ will not improve the constraints on $\Omega_{m}$ and $\Omega_{\Lambda}$ using these methods. Rather, the most important parameter is simply the total number of clusters, as long as we observe clusters with redshifts up to $z \sim 1$. This is good news because, while it is difficult to determine distances at high redshifts $(z \gtrsim 1)$ due to the low flux of these clusters in the X-ray band, recent XMM-Newton and Chandra observations readily find clusters out to redshifts of about $z=0.9$ (see for example Ettori et al. 2002). Based on our simulations we find that the combined constraints from 100 clusters uniformly distributed with an upper cut-off at $z=1$ could be used to determine $\Omega_{m}$ to within about $25 \%$, and $\Omega_{\Lambda}$ to within $20 \%(97 \%$ CL).

We show our results on combined constraints on $\Omega_{m}$ and $w$ (Model B) from simulations in Figure 2. The figure shows that the constraints from angular diameter distances alone are once again getting tighter as we choose higher redshift cut - offs, but the constraints also spread more in $w$ (they cross the $w$ axis at higher values of $w$ ). As a result, as it can be seen from this figure, the high-w limit does not improve by choosing clusters with redshift cut-offs greater than about $z \approx 1$. Using 100 clusters, the combined method could determine $w$ to about $16 \%$ (97\% CL).

We choose to study constraints on the energy densities in the Universe because they are not sensitive to systematic effects which are redshift independent. However, the energy densities are sensitive to redshift dependent systematics. Although such an effect has not yet been found, a few percent redshift dependent systematic error would be difficult to identify observationally. We carried out simulations to study how constraints on $\Omega_{m}$ and $\Omega_{\Lambda}$ (Model A) and on $\Omega_{m}$ and $w$ (Model B) change assuming redshift dependent systematics at few percent level. We show our results on Figures 3 and 4 assuming a systematic bias in the inferred distance that grows linearly from no bias at $\mathrm{z}=0$ to $\mathrm{a}+(-) 5 \%$ over(under)estimate of all distances at $\mathrm{z}=1$ using 100 clusters and $10 \%$ random errors, as before. Combining the $D_{A}$ and $d n / d z$ results still permits the measurement of the densities of matter and dark energy, or the density of matter and the equation of state parameter. Although a systematic shift in

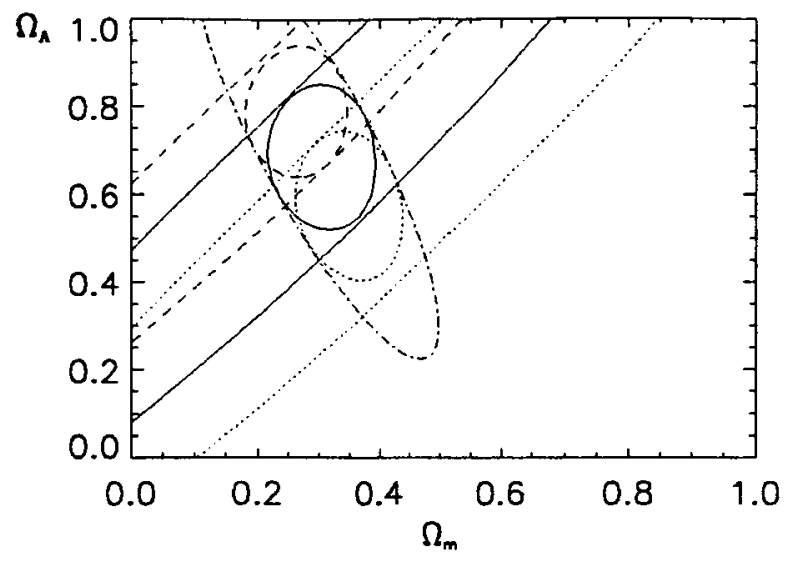

FIG. 3. - Simulated $97 \%$ confidence limits in the $\Omega_{m}-\Omega_{\Lambda}$ plane based on $d N / d z$ and $D_{A}$ measurements with redshift dependent systematic errors. The outer set of solid, dashed, and dotted lines are constraints from the $D_{A}$ values alone for a sample of 100 clusters with random errors of $10 \%$, and redshift dependent systematic errors of $+5 \%$ and $-5 \%$ at $z=1$ growing linearly from 0 at $z=0$. The dot-dashed line is the constraint from the $d N / d z$ measured in a $12 \mathrm{deg}^{2}$ SZ survey alone. The corresponding combined confidence intervals are shown as the inner solid, dashed, and dotted curves.

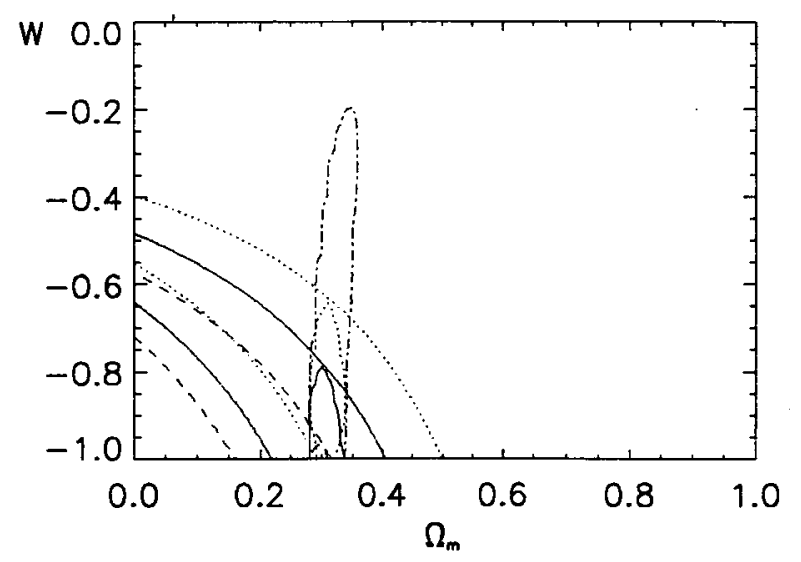

FIG. 4. - Simulated $97 \%$ confidence limits in the $\Omega_{m}-w$ plane based on $d N / d z$ and $D_{A}$ measurements with redshift dependent systematic etrors. The line codes are the same as in Figure 3.

the derived values of these parameters is caused, such 5\% systematic errors do not move the error ellipse away from the input model values significantly. While larger redshift-dependent systematic errors of this type would lead to significant systematic errors in the derived parameters, they would also inconsistencies in the $D_{A}$ and $d n / d z$ tests, and could be detected through this inconsistency.

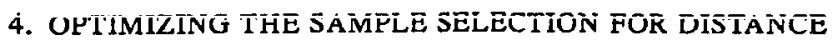 MEASUREMENTS}

Although we expect hundreds of clusters to be discovered in future X-ray and SZ surveys (for example, the XMM-Newton $64 \mathrm{deg}^{2}$ Large Scale Structure Survey will provide hundreds of candidate clusters, Refregier et al. 2002), it is not feasible to determine accurate distances to all discovered clusters. The reason for this is two-fold: (1) the observational time necessary for accurate distance determination rapidly increases with dis- 


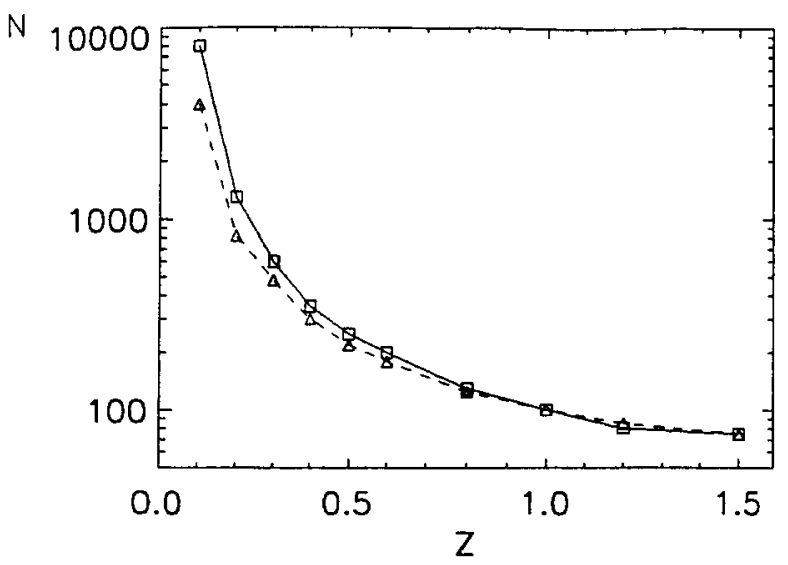

FIG. 5.- The number of clusters, $N$, in the sample for angular diameter distance determination that is necessary to achieve a fixed statistical accuracy, $25 \%$ in $\Omega_{m}, 20 \%$ in $\Omega_{\Lambda}$ (Model A) and $16 \%$ in $w$ (Model B) from combining $\mathrm{SZ}$ survey and $D_{A}$ measurements, as a function of the upper redshift cut-off of the $D_{A}$ sample. The squares and the solid curve represent the number of clusters necessary to determine $\Omega_{m}$ and $\Omega_{\Lambda}$, the triangles and the dashed curve represent the number of clusters needed to determine $w$.

tance, and is on the order of 50-100 ks with XMM-Newton and Chandra at redshifts of about 0.7 ; and (2) not all clusters are relaxed.

The question then naturally arises: How can we assemble our cluster sample optimally, yielding the most precise and robust constraints on cosmological parameters? A fundamental physical selection criterion is that the clusters should be as relaxed as possible. Based on numerical simulations, Roettiger, Stone \& Mushotzky (1997), in order to minimize the effects of dynamical activity, suggest to exclude clusters with non-cylindrical surface brightness and temperature distribution in projection, with twisted isophots, with anisotropic galaxy velocity distribution, and those clusters for which $\beta$ from spatial fitting is significantly different from $\beta$ determined from spectroscopy. If we wish to eliminate the orientation bias, we need to use clusters which are, in most part, in hydrostatic equilibrium. Although the X-ray/SZ method works for any cluster as long as we have an accurate physical model for the gas, clusters which are not in dynamical equilibrium are difficult to model, contribute a large systematic error, and so should be excluded. The signature of such objects is complicated morphology of the spatial structure in the X-ray surface brightness and/or temperature image. Line of sight mergers would be difficult to identify based on morphology. Mergers might be identified by comparing distances determined using the X-ray/SZ and other methods. The velocity distribution of member galaxies can also be used to identify line of sight merging and numerical simulations can be used to verify merging (Gomez, Hughes \& Birkinshaw 2000). A cluster which is an outlier in scaling relations (X-ray temperature - luminosity, mas s- X-ray temperature, mass - X-ray luminosity) would be a likely case of dynamical activity, since ongoing merging leads to temporary enhancements of the X-ray flux and raised average IC gas temperatures because of shock heating (Randall, Sarazin \& Ricker 2002).

In our previous simulations, we kept the number of clusters fixed at $N=100$, in order to see whether constraints improve by using clusters at $z>1$. Since it takes longer to achieve the target accuracy at higher redshifts, one has to consider the ob- servational time as well. A detailed analysis of how the required observational time for a given $D_{A}$ accuracy depends on the redshift of the cluster is beyond the scope of this paper since the necessary parameters are instrument specific. Instead we invert the question, and use simulations to determine the number of clusters necessary to observe to achieve a fixed accuracy on the cosmological parameters, as a function of the upper redshift cut-off for the $D_{A}$ sample. For concreteness, we fix the errors at the level achievable with observations of 100 clusters with $10 \%$ accuracy in the angular diameter distance determination with an upper cut-off of $z=1$, i.e. we determine the number of clusters necessary to achieve an accuracy of about $25 \%$ in $\Omega_{m}$, and $20 \%$ in $\Omega_{\Lambda}$ (for our Model A), and $16 \%$ in $w$ (for our Model B) as a function of upper redshift cut-off in the $D_{A}$ cluster sample.

In general, different numbers of clusters are necessary to achieve any specified accuracy for different parameters. In practice, we find that the ratio of errors does not change significantly in the considered redshift range and therefore once the ratio between errors in $\Omega_{m}$ and $\Omega_{\Lambda}$ is fixed, it is possible to determine the number of clusters in the sample to achieve the same ratio in accuracy in both parameters as a function of redshift cutoff. Since adding constraints from $D_{A}$ measurements to those from the redshift distribution of clusters in Model B does not improve constraints on the matter density, we determine only the number of clusters necessary to achieve a fixed accuracy in $w$ as a function of redshift. We show our results in Figure 5. Interestingly, the curves give rather similar requirements for the number of clusters. The figure also clearly shows that, as expected, with low upper cut-offs on redshifts of the sample, the necessary number of clusters increases, since the angular diameter distance is insensitive to the energy content of the Universe at low redshift. In accordance with our conclusions from Figures 1 and 2, Figure 5 shows that at redshift cutoffs $z \gtrsim 1$ the number of clusters needed tends to a fixed value. However, the observing time will increase significantly if one wants to maintain the assumed $10 \%$ accuracy in the angular diameter distance measurements. Overall, Figure 5 suggests that the optimal redshift range of a cluster sample for useful $D_{A}$ measurements is $0.5 \lesssim z \lesssim 1$.

\section{CONCLUSIONS}

We have demonstrated that the energy content of the Universe can be constrained to high statistical accuracy using the cluster redshift distribution and angular diameter distance measurements. Degeneracies in cosmological parameters that are constrained by either observable are substantially weakened when they are used in tandem. We quantified constraints from simulated cluster redshift distribution expected from a $12 \mathrm{deg}^{2} \mathrm{SZ}$ cluster survey, and constraints from simulated angular diameter distance measurements based on using the X-ray/SZ method, assuming an expected accuracy of $10 \%$ in the angular diameter distance determination of 100 clusters with redshifts $z \lesssim 1.5$. We find that $\Omega_{m}$ can be determined to a statistical accuracy ( $97 \%$ CL) of about $25 \%, \Omega_{\Lambda}$ within $20 \%$, and $w$ to an accuracy of about $16 \%$. We also addressed the question of how to select clusters of galaxies optimally for accurate diameter distance determinations. Our results indicate that the joint $d N / d z+D_{A}$ constraints on cosmological parameters for a given observation time are optimized by using cluster $D_{A}$ measurements in the redshift range $0.5 \lesssim z \lesssim 1$. We carried out simulations to study how combined constraints on the energy densities depend on redshift dependent systematic errors. We found that a com- 
bination of cluster redshift distribution and angular diameter distance determination measurements with an assumed redshift dependent systematic error which grows linearly to $5 \%$ at $z=1$ still leads to significantly improved constraints on cosmological parameters, without unacceptable systematic errors in those parameters.

Comparison of the errors derived from the $d N / d z+D_{A}$ technique with 100 clusters compares favorably with those from simulations based on type la supernovae (e.g. Gerke \& Efstathiou 2002; see also Hannestad \& Mortsell 2002; Perlmutter et al. 1999), with similar errors on $w$, but significantly tighter errors on $\Omega_{m}$. Inclusion of priors from the WMAP experiment will tighten confidence limits further (complementarity between cosmic microwave background and cluster $d N / d z$ constraints have been explicitly demonstrated in Haiman et al. 2001). We believe that the results in this paper are important, because they indicate that independent, tight statistical constraints on cosmological parameters will be available internally from galaxy cluster surveys. Having independent constraints from different methods will be invaluable in understanding the systematic errors that will likely limit our determination of cosmological parameters in the next several years.

We thank Rashid Sunyaev for advice and encouragement, Joe Mohr for valuable comments on the manuscript, and the referee for constructive comments that allowed us to improve our paper. This work (SMM) was partly supported by NASA LTSA Grant NAG5-3432 (PI: J. P. Hughes).

\section{REFERENCES}

Battye, R.A., \& Weller, J., 2003, preprint, astro-ph/0305568

Bialek, J. J., Evrand, A. E., \& Mohr, J. J. 2001, ApJ, 555, 597

Birkinshaw, M., \& Hughes, J. P., 1994, ApJ, 420, 33

Bridle, S. L., Lahav, O., Ostriker, J. P., \& Steinhardt, P. J. 2003, Science, 299, 1532

Carlstrom, J. E. , Holder, G. P., \& Reese, E. D., 2002, ARA\&A, 40, 643

Cash, W. 1979, ApJ, 228, 939

Cavaliere, A., \& Fusco-Femiano, R., 1976, A\&A, 49, 137

Ettori, S., Tozzi, P., \& Rosati, P., ApJ, submitted, astro-ph/0211335

Evrard et al. 2002, ApJ, 573, 7

Fox, D.C. \& Pen, U., 2002, ApJ, 574, 38

Gerke, B. F., \& Efstathiou, G. 2002, MNRAS, 335, 33

Gomez, P.L., Hughes, J.P. \& Birkinshaw, M., 2000, ApJ, 540, 726

Haiman, Z., Mohr, J. J., \& Holder, G. P. 2001, ApJ, 553, 545

Hannestad, S., \& Mortsell, E. 2002, PRD 66, 063508

Holder, G. P., Haiman, Z., \& Mohr, J. J. 2001, ApЛ, 560, 111

Holzapfel, W. L., et al. 1997, ApJ, 480, 449

Hu, W. 2003, Physical Review D, , submitted, astro-ph/0301416

Hu \& Kravtsov 2003, ApJ, 584, 702

Inagaki, Y., Suginohara, T., \& Suto, Y., 1995, PASJ, 47, 411

Jones, C., \& Forman, W., 1984, ApJ, 276, 38

Kujat, J., Linn, A.M., Scherrer, R.J., \& Weinberg, D.H., 2002, ApJ, 572, 1

Levine, E. S., Schulz, A. E., \& White, M. 2002, ApJ, 577,569

Majumdar, S., \& Mohr, J. J. 2003, ApJ, 585, 603

Mathiesen, B., Evrard, A.E., \& Mohr, J.J., 1999, ApJ, 520, L21

Mei, S., \& Bartlett, J. G. 2003, A\&A, submitted, astro-ph/0302567

Mohr, J. J., Mathiesen, B., \& Evrard, A. E., 1999, ApJ, 517, 627

Molnar, S. M., Birkinshaw, M., \& Mushotzky, R. F. 2002, ApJ, 570, 1

Newman, J.A.; Marinoni, C., Coil, A.L., \& Davis, M., 2002, YAS̄Y, i î4, ẑy

Perlmutter, S., et al., 1999, ApJ, 517, 565

Pratt, G. W.; Amaud, M., 2002, A\&A, 394, 375

Randall, S.W., Sarazin, C.L., \& Ricker, P.M., 2002, ApJ, 577, 579

Reese, E. D., Carlstrom, J. E., Joy, M., Mohr, J. J., Grego, L., \& Holzapfel, W. L., 2003, ApJ, 581, 53

Refregier, A., Valtchanov, I., \& Pierre, M. 2002, A\&A, 390, 1

Roettiger, K., Stone, J. M., \& Mushotzky, R. F., 1997, ApJ, 482, 588

Rosati, P., Borgani, S., \& Norman, C. 2002, ARA\&A, 40, 539

Rubino-Martin, J. A., \& Sunyaev, R. A. 2002, MNRAS, submitted, astro$\mathrm{ph} / 0211430$

Spergel, D. N. et al. 2003, ApJ, submitted, astro-ph/0302209

Sinvam R A \& 7al'dhwich Y $R$ 1080 ARA\&A 18537
Verde, L., Haiman, Z., \& Spergel, D. N. 2002, ApJ, 581, 5

Vikhlinin, A. et al. 2003, ApJ, in press, astro-ph/0212075

Wang, L., \& Steinhardt, P. J. 1998, ApJ, 508, 483

Weller, J., Battye, R. A., \& Kneissl, R., 2002, Physical Review Letters, 88, Issue 23, id. 231301

Weller, J., \& Albrecht, A., 2002, Physical Review D, 65, Issue 10, id. 103512 